\title{
Normality of the p-Harmonic and Log-p-Harmonic Mappings
}

\author{
Qinghua Jiang, Fanning Meng $\mathbb{D}$, and Guoqiang Dang $\mathbb{1}$ \\ School of Mathematical and Information Science, Guangzhou University, 510006 Guangzhou, China \\ Correspondence should be addressed to Fanning Meng; mfnfdbx@163.com and Guoqiang Dang; dangguoqiang@126.com
}

Received 16 June 2020; Revised 21 August 2020; Accepted 7 October 2020; Published 6 November 2020

Academic Editor: Liguang Wang

Copyright ( 2020 Qinghua Jiang et al. This is an open access article distributed under the Creative Commons Attribution License, which permits unrestricted use, distribution, and reproduction in any medium, provided the original work is properly cited.

In this paper, the concepts of p-harmonic mappings and log-p-harmonic mappings in the unit disk have been introduced and studied by many researchers. We proved the normality of the p-harmonic mappings and log-p-harmonic mappings, which extend the related results of harmonic mappings of earlier authors.

\section{Introduction and Preliminaries}

For real-valued harmonic functions defined in $\mathbb{D}$, Lappan [1] established that $\varphi$ is normal if

$$
\sup _{z \in \mathbb{D}}\left(1-|z|^{2}\right) \frac{|\operatorname{grad} \varphi(z)|}{1+\varphi^{2}(z)}<\infty,
$$

where $\operatorname{grad} \varphi$ is the gradient vector of $\varphi$. In [2], the authors also proved geometric properties of real-valued harmonic normal functions. Namely, a real-valued harmonic function $\varphi$ with the property

$$
\iint_{\mathbb{D}}\left(\frac{|\operatorname{grad} \varphi(z)|}{1+\varphi^{2}(z)}\right)^{2} d \Omega<\infty,
$$

is normal.

Recently, many authors considered the properties of the complex-valued harmonic mappings and harmonic quasiconformal mappings in [3-13]. We are motivated to establish the topic of normality for complex-valued p-harmonic mappings and log-p-harmonic mappings defined in the unit disk. An important concept related with normal harmonic functions is the Bloch function, which was studied by Colonna in [14]. It is a classical result of Lewy [15] that a harmonic mapping is locally univalent in a domain $\Omega$ if and only if its Jacobian does not vanish. In terms of the canonical decomposition, the Jacobian of harmonic mappings $f=h+\bar{g}$ is given by $J_{f}=\left|h^{\prime}\right|^{2}-\left|g^{\prime}\right|^{2}$, and thus, a locally univalent har- monic mapping in a simply connected domain $\Omega$ will be sense-preserving if $\left|h^{\prime}\right|>\left|g^{\prime}\right|$.

Following the above ideas, particularly the definition of Bloch harmonic function given by Colonna [14], we will prove that the polyharmonic mapping $F$ and log-pharmonic mapping $f$ defined in the unit disk $\mathbb{D}$ are normal if they satisfy a Lipschitz type condition. Further, for the complex-valued polyharmonic mappings and log-pharmonic mappings, we give out some additional conditions for which are normal. These conditions cannot be omitted. A 2 p-times continuously differentiable complex-valued function $F(z)=u(z)+i v(z)$ in a domain $D \subseteq \mathbb{C}$ is polyharmonic mapping or $\mathrm{p}$-harmonic if $F(z)$ satisfies the $\mathrm{p}$-harmonic equation

$$
\Delta^{p} F=\Delta\left(\Delta^{p-1}\right) F=0,
$$

where the Laplacian operator

$$
\Delta F=4 F_{z \bar{z}}=\frac{\partial^{2} F}{\partial x^{2}}+\frac{\partial^{2} F}{\partial y^{2}} .
$$

As we see in Proposition 1 in [16], we know that a mapping $F$ is polyharmonic in a simply connected domain $D \subseteq \mathbb{C}$ if and only if $F$ has the following representation

$$
F(z)=\sum_{k=1}^{p}|z|^{2(k-1)} G_{p-k+1}(z),
$$


where each $G_{p-k+1}$ is harmonic for $k \in\{1, \cdots, p\}$. When $p=1$, the mapping $F$ is called harmonic. When $p=2$, the mapping $F$ is called biharmonic. $f$ is called log-p-harmonic mapping if $\log f$ is p-harmonic mapping. When $p=1$, the mapping $f$ is called log-harmonic. When $p=2$, the mapping $f$ is called log-biharmonic, which can be regarded as generalizations of holomorphic functions. So we say that $f$ is called log-pharmonic mapping in a simply connected domain $D \subseteq \mathbb{C}$ if and only if $f$ has the form

$$
f(z)=\prod_{k=1}^{p}\left[g_{p-k+1}(z)\right]^{|z|^{2(k-1)}}
$$

where each $g_{p-k+1}$ is log-harmonic for $k \in\{1, \cdots, p\}$.

For a continuously differentiable mapping $f$ in $D$, we define

$$
\begin{aligned}
& \Lambda_{f}(z)=\max _{0 \leq \theta \leq 2 \pi}\left|f_{z}(z)+\exp (-2 i \theta) f_{\bar{z}}(z)\right|=\left|f_{z}(z)\right|+\left|f_{\bar{z}}(z)\right|, \\
& \lambda_{f}(z)=\min _{0 \leq \theta \leq 2 \pi}\left|f_{z}(z)+\exp (-2 i \theta) f_{\bar{z}}(z)\right|=|| f_{z}(z)|-| f_{\bar{z}}(z) \| .
\end{aligned}
$$

Recently, many authors considered Landau-type theorems for harmonic mappings, biharmonic mappings, and p-harmonic mappings [16-23]. Li and Wang [24] introduced the log-p-harmonic mappings and derived two versions of Landau-type theorems. However, in virtue of being inspired by these results, we establish the normality of polyharmonic mappings and log-p-harmonic mappings.

\section{Necessary Lemmas}

In order to derive our main results, we need the following lemmas.

Lemma 1. [14]. Suppose that $f(z)=h(z)+g \overline{(z})$ is a harmonic mapping of the unit disk $\mathbb{D}$ with $h(z)=\sum_{n=1}^{\infty} a_{n} z^{n}$ and $g(z)$ $=\sum_{n=1}^{\infty} b_{n} z^{n}$ are analytic on $\mathbb{D}$. If $|f(z)|<M$ for all $z \in \mathbb{D}$, then

$$
\Lambda_{f}(z) \leq \frac{4 M}{\pi\left(1-|z|^{2}\right)}
$$

Lemma 2. [22]. Suppose that $f(z)=h(z)+g \overline{(z)}$ is a harmonic mapping of the unit disk $\mathbb{D}$ with $h(z)=\sum_{n=1}^{\infty} a_{n} z^{n}$ and $g(z)$ $=\sum_{n=1}^{\infty} b_{n} z^{n}$ are analytic on $\mathbb{D}$. If $|f(z)|<M$ for all $z \in \mathbb{D}$, then for $|z|=r<1$, we have

$$
|f(z)| \leq \frac{4 M r}{\pi(1-r)}
$$

Lemma 3. [25]. Suppose that $f(z)=h(z)+g \overline{(z})$ is a harmonic mapping of the unit disk $\mathbb{D}$ with $h(z)=\sum_{n=1}^{\infty} a_{n} z^{n}$ and $g(z)$ $=\sum_{n=1}^{\infty} b_{n} z^{n}$ are analytic on $\mathbb{D}$ and $\lambda_{f}(0)=1$. If $\Lambda_{f}(z) \leq \Lambda$ for all $z \in \mathbb{D}$, then

$$
\left|a_{n}\right|+\left|b_{n}\right| \leq \frac{\Lambda^{2}-1}{n \Lambda}, \quad n=2,3, \cdots
$$

When $\Lambda>1$, the above estimates are sharp for all $n=2$, $3, \cdots$, with the extremal functions $f_{n}(z)$ and $f_{n}(z)$ as follows

$$
f_{n}(z)=\Lambda^{2} z-\left(\Lambda^{3}-\Lambda\right) \int_{0}^{z} \frac{1}{\Lambda+z^{n-1}} d z .
$$

When $\Lambda=1$, then $f(z)=a_{1} z+\overline{b_{1}} \bar{z}$ with $\| a_{1}|-| b_{1}||=1$.

Lemma 4. [19]. Suppose that $f(z)=h(z)+g \overline{(z)}$ is a harmonic mapping of the unit disk $\mathbb{D}$ with $h(z)=\sum_{n=1}^{\infty} a_{n} z^{n}$ and $g(z)$ $=\sum_{n=1}^{\infty} b_{n} z^{n}$ are analytic on $\mathbb{D}$. If $\Lambda_{f}(z) \leq \Lambda$ for all $z \in \mathbb{D}$, then for each $z \in \mathbb{D}$,

$$
|f(z)| \leq \Lambda z .
$$

We recall that the chordal distance on the generalized complex plane $\widehat{\mathbb{C}}$, which is defined by

$$
\begin{aligned}
\chi\left(z_{1}, z_{2}\right) & =\frac{\left|z_{1}-z_{2}\right|}{\sqrt{1+\left|z_{1}\right|^{2}} \sqrt{1+\left|z_{2}\right|^{2}}} ; z_{1}, z_{2} \in \mathbb{C}, \\
\chi(z, \infty) & =\frac{1}{\sqrt{1+|z|^{2}}} .
\end{aligned}
$$

If $P_{z_{1}}, P_{z_{2}}$ are the two points on the Riemann sphere, under stereographic projection, corresponding to $z_{1}$ and $z_{2}$, respectively, we have

$$
\left|P_{z_{1}}-P_{z_{2}}\right|=\chi\left(z_{1}, z_{2}\right)
$$

Therefore,

$$
L(\Gamma) \geq \rho\left(z_{1}, z_{2}\right) \geq \chi\left(z_{1}, z_{2}\right)
$$

where $\mathrm{Q}\left(z_{1}, z_{2}\right)$ is the spherical distance of $z_{1}$ and $z_{2}, \Gamma$ is any rectifiable curve in $\mathbb{C}$ with endpoints $z_{1}, z_{2}$, and

$$
L(\Gamma)=\int_{\Gamma} \frac{|d u|}{1+|u|^{2}}
$$

is the spherical length of $\Gamma$. On the basis of the paper, given $z_{1}, z_{2} \in \mathbb{D}, \rho\left(z_{1}, z_{2}\right)$ denotes the hyperbolic distance between $z_{1}, z_{2}$. Therefore, if $\tau$ denotes the hyperbolic geodesic joining $z_{1}$ to $z_{2}$, then

$$
\rho\left(z_{1}, z_{2}\right)=\int_{\tau} \frac{|d v|}{1-|v|^{2}}
$$

More explicitly,

$$
\rho\left(z_{1}, z_{2}\right)=\frac{1}{2} \log \frac{1+\lambda}{1-\lambda},
$$


where

$$
\lambda=\left|\frac{z_{1}-z_{2}}{1-\bar{z}_{1} z_{2}}\right| .
$$

With these notations, a polyharmonic mapping or log-pharmonic mapping $f: \mathbb{D} \rightarrow \mathbb{C}$ is called a normal polyharmonic mapping or normal log-p-harmonic mapping, if

$$
\sup _{z_{1} \neq z_{2}} \frac{\chi\left(f\left(z_{1}\right), f\left(z_{2}\right)\right)}{\rho\left(z_{1}, z_{2}\right)}<\infty
$$

The following lemma provides an alternative method for deciding when a polyharmonic mapping or log-p-harmonic mapping is normal.

Lemma 5. Let $f(z)$ be a polyharmonic mapping or log-pharmonic mapping in the unit disk $\mathbb{D}$, then $f$ is normal if

$$
\|f\|:=\sup _{z \in \mathbb{D}}\left(1-|z|^{2}\right) \frac{\left|f_{z}\right|+\left|f_{\bar{z}}\right|}{1+|f|^{2}}<\infty .
$$

Proof. Suppose that $\|f\|<\infty$ and let $z_{1}, z_{2} \in \mathbb{D}$. If $\tau:[0,1] \rightarrow \mathbb{D}$ is the hyperbolic geodesic with endpoints $z_{1}$ and $z_{2}$,

$$
\chi\left(f\left(z_{1}\right), f\left(z_{2}\right)\right) \leq \int_{f_{\circ} \tau} \frac{|d u|}{1+|u|^{2}}=\int_{0}^{1} \frac{\left|d f(\tau(t)) \tau^{\prime}(t)\right|}{1+|f(\tau(t))|^{2}} d t,
$$

where $d f$ stands for the differential of $f$. From here and (21), we have

$$
\begin{aligned}
\chi\left(f\left(z_{1}\right), f\left(z_{2}\right)\right) & \leq \int_{0}^{1} \frac{\left|d f(\tau(t)) \tau^{\prime}(t)\right|}{1+|f(\tau(t))|^{2}} d t \\
& \leq \int_{0}^{1} \sup _{z \in \mathbb{D}} \frac{1-|z|^{2}}{1-|\tau(t)|^{2}} \frac{\left|d f(\tau(t)) \| \tau^{\prime}(t)\right|}{1+|f(\tau(t))|^{2}} d t \\
& =\int_{0}^{1}\left\{\sup _{z \in \mathbb{D}}\left(1-|z|^{2}\right) \frac{|d f(\tau(t))|}{1+|f(\tau(t))|^{2}}\right\} \frac{\left|\tau^{\prime}(t)\right|}{1-|\tau(t)|^{2}} \\
& \leq\|f\| \cdot \int_{0}^{1} \frac{\left|\tau^{\prime}(t)\right|}{1-|\tau(t)|^{2}} d t=\|f\| \cdot \rho\left(z_{1}, z_{2}\right) .
\end{aligned}
$$

Hence, we obtain

$$
\sup _{z_{1} \neq z_{2}} \frac{\chi\left(f\left(z_{1}\right), f\left(z_{2}\right)\right)}{\rho\left(z_{1}, z_{2}\right)}<\infty
$$

So it implies that $f$ is normal.

\section{Main Results and Their Proofs}

In this section, we prove the normality of the polyharmonic mappings and log-p-harmonic mappings as follows.
Theorem 6. Let $F(z)=\sum_{k=1}^{p}|z|^{2(k-1)} G_{p-k+1}(z)$ be a polyharmonic mapping in the unit disk $\mathbb{D}$ satisfying $F(0)=\lambda_{F}(0)$ $1=0$. Suppose that for each $k \in\{1, \cdots, p\}$, we have

$$
G_{p-k+1}(z) \text { is harmonic in } \mathbb{D} \text {, and } G_{p-k+1}(0)=0 \text {; }
$$

$\left|G_{p-k+1}(z)\right| \leq M_{p-k+1}$, and $\Lambda_{G_{p}}(z) \leq \Lambda_{p}$, where $M_{p-k+1} \geq 0, \Lambda_{p} \geq 1$.

Then, $F$ is normal polyharmonic mapping of the unit disk $\mathbb{D}$.

Proof. We may represent the harmonic functions $G_{p-k+1}(z)$ in series form as

$$
\begin{aligned}
G_{p-k+1}(z)= & \sum_{j=1}^{\infty} a_{j, p-k+1} z^{j}+\sum_{j=1}^{\infty} b_{j, p k+1}^{-} \bar{z}^{j}, k \in\{1,2, \cdots, p\} ; \\
& \left\|a_{1, p}|-| b_{1, p}\right\|=\lambda_{F}(0)=\left\|\left(G_{p}\right)_{z}(0)|-|\left(G_{p}\right)_{\bar{z}}(0)\right\| \\
= & \lambda_{G_{p}}(0)=1,\left|a_{1, p}\right|+\left|b_{1, p}\right|=\left|\left(G_{p}\right)_{z}(0)\right|+\left|\left(G_{p}\right)_{\bar{z}}(0)\right| \\
= & \Lambda_{G_{p}}(0) \leq \Lambda_{p} .
\end{aligned}
$$

Firstly, we calculate the boundedness of the derivative of $F$.

$$
\begin{aligned}
\mid F_{z}(z)+ & F_{\bar{z}}(z)|\leq| F_{z}(z)|+| F_{\bar{z}}(z) \mid \\
= & \left|\left(G_{p}\right)_{z}(z)+\sum_{k=1}^{p-1}\left[|z|^{2 k}\left(G_{p-k}\right)_{z}(z)+k G_{p-k}(z) \bar{z}^{k} z^{k-1}\right]\right| \\
& +\left|\left(G_{p}\right)_{\bar{z}}(z)+\sum_{k=1}^{p-1}\left[|z|^{2 k}\left(G_{p-k}\right)_{\bar{z}}(z)+k G_{p-k}(z) \bar{z}^{k-1} z^{k}\right]\right| \\
\leq & A_{1}+A_{2}+A_{3}+A_{4},
\end{aligned}
$$

where

$$
\begin{aligned}
& A_{1}=\left|\left(G_{p}\right)_{z}(0)+\left(G_{p}\right)_{\bar{z}}(0)\right|, \\
& A_{2}=\left.\left|\sum_{k=1}^{p-1}\right| z\right|^{2 k}\left[\left(G_{p-k}\right)_{z}(z)+\left(G_{p-k}\right)_{\bar{z}}(z)\right] \mid, \\
& A_{3}=\left|\sum_{k=1}^{p-1} k G_{p-k}(z)\left(\bar{z}^{k} z^{k-1}+\bar{z}^{k-1} z^{k}\right)\right|, \\
& A_{4}=\left|\left[\left(G_{p}\right)_{z}(z)-\left(G_{p}\right)_{z}(0)\right]+\left[\left(G_{p}\right)_{\bar{z}}(z)-\left(G_{p}\right)_{\bar{z}}(0)\right]\right| .
\end{aligned}
$$

By a simple calculation, we have

$$
A_{1} \leq \Lambda_{G_{p}}(0) \leq \Lambda_{p}
$$


Using Lemma 1, we have

$$
\begin{aligned}
A_{2} & \leq \sum_{k=1}^{p-1}|z|^{2 k}\left[\left|\left(G_{p-k}\right)_{z}(z)\right|+\left|\left(G_{p-k}\right)_{\bar{z}}(z)\right|\right] \leq \sum_{k=1}^{p-1} r^{2 k} \Lambda_{G_{p-k}}(z) \\
& \leq \sum_{k=1}^{p-1} r^{2 k} \frac{4 M_{p-k}}{\pi\left(1-r^{2}\right)} .
\end{aligned}
$$

By Lemma 2, we have

$$
A_{3} \leq \sum_{k=1}^{p-1}\left|k G_{p-k}(z)\right|\left(\left|\bar{z}^{k} z^{k-1}\right|+\left|\bar{z}^{k-1} z^{k}\right|\right) \leq \frac{8}{\pi(1-r)} \sum_{k=1}^{p-1} k M_{p-k} r^{2 k} .
$$

Using Lemma 3, we have

$$
\begin{aligned}
A_{4} & \leq\left|\left(G_{p}\right)_{z}(z)-\left(G_{p}\right)_{z}(0)\right|+\left|\left(G_{p}\right)_{\bar{z}}(z)-\left(G_{p}\right)_{\bar{z}}(0)\right| \\
& \leq \sum_{n=2}^{\infty}\left(\left|a_{n, p}\right|+\left|b_{n, p}\right|\right) n r^{n-1} \\
& \leq \sum_{n=2}^{\infty} \frac{\left(\Lambda_{p}^{2}-1\right) r^{n-1}}{\Lambda_{p}}=\frac{\Lambda_{p}^{2}-1}{\Lambda_{p}} \frac{r}{1-r} .
\end{aligned}
$$

By the above estimates, we obtain the following result

$$
\left|F_{z}(z)+F_{\bar{z}}(z)\right| \leq S_{1}(r)
$$

where

$$
\begin{aligned}
S_{1}(r)= & \frac{4}{\pi\left(1-r^{2}\right)} \sum_{k=1}^{p-1} M_{p-k} r^{2 k}+\frac{8}{\pi(1-r)} \sum_{k=1}^{p-1} k M_{p-k} r^{2 k} \\
& +\frac{\left(\Lambda_{p}^{2}-1\right) r}{\Lambda_{p}(1-r)}+\Lambda_{p} .
\end{aligned}
$$

Now, differentiating $S_{1}(r)$, we have

$$
\begin{aligned}
S_{1}^{\prime}(r)= & \sum_{k=1}^{p-1} \frac{4 M_{p-k}\left[2 k r^{2 k-1}\left(1-r^{2}\right)+2 r^{2 k+1}\right]}{\pi\left(1-r^{2}\right)^{2}} \\
& +\sum_{k=1}^{p-1} \frac{8 k M_{p-k}\left[2 k r^{2 k+1}(1-r)+r^{2 k}\right]}{\pi(1-r)^{2}}+\frac{\Lambda_{p}^{2}-1}{\Lambda_{p}(1-r)^{2}} .
\end{aligned}
$$

In view of $\Lambda_{p} \geq 1$ and $r \in(0,1)$, after a simple calculation, it shows that $S_{1}^{\prime}(r)>0$. It is simple to verify that $S_{1}(r)$ is strictly increasing in $(0,1)$.

$$
\lim _{r \rightarrow 0} S_{1}(r)=\Lambda_{p}, \lim _{r \rightarrow 1} S_{1}(r)=+\infty .
$$

Obviously, $S_{1}(r)$ has only one pole $r=1(|z|=r)$. In other words, $S_{1}(r)$ is bounded in the interval $(0,1)$.

Finally, we consider the boundedness of $F$ for any $|z|=r_{1}$, then we have

$$
\begin{aligned}
|F(z)|= & \left.\left|\sum_{n=1}^{\infty}\left(a_{n, p} z^{n}+b_{n, p}^{-} \bar{z}^{n}\right)+\sum_{k=1}^{p-1}\right| z\right|^{2 k} G_{p-k}(z) \mid \\
\leq & \left|a_{1, p} z+b_{1, p}^{-} \bar{z}\right|+\left|\sum_{n=2}^{\infty}\left(a_{n, p} z^{n}+b_{n, p}^{-} \bar{z}^{n}\right)\right| \\
& +\left.\left|\sum_{k=1}^{p-1}\right| z\right|^{2 k} G_{p-k}(z) \mid \leq \Lambda_{p} r_{1}+\frac{\Lambda_{p}^{2}-1}{\Lambda_{p}} \sum_{n=2}^{\infty} \frac{r_{1}^{n}}{n} \\
& +\sum_{k=1}^{p-1} r_{1}^{2 k} \frac{4 M_{p-k} r_{1}}{\pi\left(1-r_{1}\right)}=\Lambda_{p} r_{1}-\frac{\Lambda_{p}^{2}-1}{\Lambda_{p}}\left[r_{1}+\ln \left(1-r_{1}\right)\right] \\
& +\frac{4 r_{1}}{\pi\left(1-r_{1}\right)} \sum_{k=1}^{p-1} M_{p-k} r_{1}^{2 k} .
\end{aligned}
$$

So $|F(z)| \leq S_{2}\left(r_{1}\right)$, where

$S_{2}\left(r_{1}\right)=\Lambda_{p} r_{1}-\frac{\Lambda_{p}^{2}-1}{\Lambda_{p}}\left[r_{1}+\ln \left(1-r_{1}\right)\right]+\frac{4 r_{1}}{\pi\left(1-r_{1}\right)} \sum_{k=1}^{p-1} M_{p-k} r_{1}^{2 k}$.

By the similar approach for differentiating $S_{2}\left(r_{1}\right)$, we have the following one

$$
\begin{aligned}
S_{2}^{\prime}\left(r_{1}\right)= & \sum_{k=1}^{p-1} \frac{4 M_{p-k}\left[(2 k+1) r_{1}^{2 k}\left(1-r_{1}\right)+r_{1}^{2 k+1}\right]}{\pi\left(1-r_{1}\right)^{2}} \\
& +\frac{\Lambda_{p}^{2}-1}{\Lambda_{p}^{2}\left(1-r_{1}\right)}+\frac{1}{\Lambda_{p}}
\end{aligned}
$$

By elementary calculations, we get $S_{2}{ }^{\prime}\left(r_{1}\right)>0$. It implies that $S_{2}\left(r_{1}\right)$ is increasing for $\Lambda_{p} \geq 1$ and $r_{1} \in(0,1)$. It is simple to verify that $S_{2}\left(r_{1}\right)$ is bounded in $(0,1)$. Combined with Lemma 5 and Estimation (33), we conclude ultimately that $F$ is normal polyharmonic mapping in the unit disk $\mathbb{D}$. The proof of this theorem is complete.

Theorem 7. Let $F(z)=\sum_{k=1}^{p}|z|^{2(k-1)} G_{p-k+1}(z)$, be a polyharmonic mapping of $\mathbb{D}$ satisfying $F(0)=\lambda_{F}(0)-1=0$. Suppose that for $k \in\{1,2, \cdots, p\}$, we have

$$
\begin{gathered}
G_{p-k+1}(z) \text { is harmonic in } \mathbb{D} \text {, and } G_{p-k+1}(0)=0 ; \\
\begin{aligned}
\Lambda_{G_{p-k+1}}(z) & \leq \Lambda_{p-k+1} \text { for all } z \in \mathbb{D} \text {, where } \Lambda_{p-k+1} \geq 0, k \\
& =2,3, \cdots, p \text {, and } \Lambda_{p} \geq 1 .
\end{aligned}
\end{gathered}
$$

Then, $F$ is normal polyharmonic mapping in the unit disk $\mathbb{D}$. 
Proof. We represent the harmonic functions $G_{p-k+1}(z)$ in series form as

$$
G_{p-k+1}(z)=\sum_{j=1}^{\infty} a_{j, p-k+1} z^{j}+\sum_{j=1}^{\infty} b_{j, p k+1}^{-} \bar{z}^{j}
$$

for each $k \in\{1,2, \cdots, p\}$, and

$$
\left|a_{1, p}\right|+\left|b_{1, p}\right|=\Lambda_{F}(0)=\left|\left(G_{p}\right)_{z}(0)\right|+\left|\left(G_{p}\right)_{\bar{z}}(0)\right|=\Lambda_{G_{p}}(0) \leq \Lambda_{p},
$$$$
\left\|a_{1, p}|-| b_{1, p}||=\lambda_{F}(0)=\right\|\left(G_{p}\right)_{z}(0)|-|\left(G_{p}\right)_{\bar{z}}(0) \|=\lambda_{G_{p}}(0)=1
$$

To prove the normality of $F$, we first determine that the derivative of $F$ is bounded in $\mathbb{D}$. Then, as in the proof of Theorem 6 , we have

$$
\left|F_{z}(z)+F_{\bar{z}}(z)\right| \leq B_{1}+B_{2}+B_{3}+B_{4}
$$

where

$$
\begin{aligned}
B_{1} & \leq\left|\left(G_{p}\right)_{z}(0)\right|+\left|\left(G_{p}\right)_{\bar{z}}(0)\right|=\Lambda_{G_{p}}(0) \leq \Lambda_{p}, \\
B_{2} & \leq \sum_{k=1}^{p-1}|z|^{2 k}\left[\left|\left(G_{p-k}\right)_{z}(z)\right|+\left|\left(G_{p-k}\right)_{\bar{z}}(z)\right|\right] \\
& \leq \sum_{k=1}^{p-1} r^{2 k} \Lambda_{G_{p-k}}(z) \leq \sum_{k=1}^{p-1} \Lambda_{p-k} r^{2 k} .
\end{aligned}
$$

By the condition (2) of Theorem 7 and Lemma 4, we have

$$
B_{3} \leq \sum_{k=1}^{p-1}\left|k G_{p-k}(z)\right|\left(\left|\bar{z}^{k} z^{k-1}\right|+\left|\bar{z}^{k-1} z^{k}\right|\right) \leq \sum_{k=1}^{p-1} 2 k \Lambda_{p-k} r^{2 k}
$$

Applying Lemma 3, we have

$$
\begin{aligned}
B_{4} & \leq\left|\left(G_{p}\right)_{z}(z)-\left(G_{p}\right)_{z}(0)\right|+\left|\left(G_{p}\right)_{\bar{z}}(z)-\left(G_{p}\right)_{\bar{z}}(0)\right| \\
& \leq \sum_{n=2}^{\infty} n\left(\left|a_{n, p}\right|+\left|b_{n, p}\right|\right) r^{n-1} \leq \frac{\Lambda_{p}^{2}-1}{\Lambda_{p}} \sum_{n=2}^{\infty} r^{n-1} \\
& =\frac{\Lambda_{p}^{2}-1}{\Lambda_{p}} \frac{r}{1-r} .
\end{aligned}
$$

By above estimates, we obtain that

$$
\begin{aligned}
\left|F_{z}(z)+F_{\bar{z}}(z)\right| \leq & \sum_{k=1}^{p-1} \Lambda_{p-k} r^{2 k}+\sum_{k=1}^{p-1} 2 k \Lambda_{p-k} r^{2 k} \\
& +\frac{\Lambda_{p}^{2}-1}{\Lambda_{p}} \frac{r}{1-r}+\Lambda_{p} \\
= & \sum_{k=1}^{p-1}(2 k+1) \Lambda_{p-k} r^{2 k}+\frac{\Lambda_{p}^{2}-1}{\Lambda_{p}} \frac{r}{1-r}+\Lambda_{p} .
\end{aligned}
$$

Set

$$
S_{3}(r)=\sum_{k=1}^{p-1}(2 k+1) \Lambda_{p-k} r^{2 k}+\frac{\Lambda_{p}^{2}-1}{\Lambda_{p}} \frac{r}{1-r}+\Lambda_{p}
$$

For differentiating $S_{3}(r)$, we have

$$
S_{3}{ }^{\prime}(r)=\sum_{k=1}^{p-1} 2 k(2 k+1) \Lambda_{p-k} r^{2 k-1}+\frac{\Lambda_{p}^{2}-1}{\Lambda_{p}(1-r)^{2}} .
$$

By a simple calculation, we obtain $S_{3}{ }^{\prime}(r)>0$. It shows that $S_{3}(r)$ is strictly increasing in $r \in(0,1)$, and

$$
\lim _{r \rightarrow 0} S_{3}(r)=\Lambda_{p}, \lim _{r \rightarrow 1} S_{3}(r)=+\infty
$$

This shows that $S_{3}(r)$ has only one pole $r=1$, and it says that $S_{3}(r)$ is bounded in $(0,1)$. So $\left|F_{z}(z)+F_{\bar{z}}(z)\right|$ is a finite value in the unit disk $\mathbb{D}$.

Now, we consider any $z$ with $|z|=r_{2}$. Then, we have

$$
\begin{aligned}
|F(z)|= & \left.\left|\sum_{n=1}^{\infty}\left(a_{n, p} z^{n}+b_{n, p}^{-} \bar{z}^{n}\right)+\sum_{k=1}^{p-1}\right| z\right|^{2 k} G_{p-k}(z) \mid \\
\leq & \left|a_{1, p} z+b_{1, p}^{-} \bar{z}\right|+\left|\sum_{n=2}^{\infty}\left(a_{n, p} z^{n}+b_{n, p}^{-} \bar{z}^{n}\right)\right| \\
& +\left.\left|\sum_{k=1}^{p-1}\right| z\right|^{2 k} G_{p-k}(z) \mid \leq \Lambda_{p} r_{2}+\frac{\Lambda_{p}^{2}-1}{\Lambda_{p}} \sum_{n=2}^{\infty} \frac{r_{2}^{n}}{n} \\
& +\sum_{k=1}^{p-1} \Lambda_{p-k} r_{2}^{2 k+1}=\sum_{k=1}^{p-1} \Lambda_{p-k} r_{2}^{2 k+1} \\
& -\frac{\Lambda_{p}^{2}-1}{\Lambda_{p}}\left[r_{2}+\ln \left(1-r_{2}\right)\right]+\Lambda_{p} r_{2} .:=S_{4}\left(r_{2}\right)
\end{aligned}
$$

Differentiating $S_{4}\left(r_{2}\right)$, we have

$$
S_{4}{ }^{\prime}\left(r_{2}\right)=\sum_{k=1}^{p-1}(2 k+1) \Lambda_{p-k} r^{2 k}+\frac{\Lambda_{p}^{2}-1}{\Lambda_{p}\left(1-r_{2}\right)}+\frac{1}{\Lambda_{p}}
$$

After elementary calculations, we have that $S_{4}{ }^{\prime}\left(r_{2}\right)>0$. It implies that $S_{4}\left(r_{2}\right)$ is strictly increasing. It is simple to verify that $S_{4}\left(r_{2}\right)$ is finite for $r_{2} \in(0,1)$. It says that $F$ is bounded in $\mathbb{D}$. Using these above estimates and Condition (21), we 
conclude that $F$ is normal polyharmonic mapping in $\mathbb{D}$. This proof of Theorem 7 is complete.

Finally, we establish the normality of log-p-harmonic mappings as follows.

Theorem 8. Let $f(z)=\prod_{k=1}^{p}\left[g_{p-k+1}(z)\right]^{|z|^{2(k-1)}}$ be a log-p-harmonic mapping in the unit disk $\mathbb{D}$ satisfying $f(0)=g_{p}(0)=$ $\lambda_{f}(0)=1$. Suppose that for each $k \in\{1, \cdots, p\}$ we have

$$
\begin{gathered}
g_{p-k+1}(z) \text { is } \log -\text { harmonic in } \mathbb{D} \\
\left|g_{p-k+1}(z)\right| \leq M_{p-k+1}, \text { let } G_{p}=\log g_{p}, \Lambda_{G_{p}} \\
\leq \Lambda_{p}, \text { where } M_{p-k+1} \geq 1, \Lambda_{p} \geq 1
\end{gathered}
$$

Then, $f$ is a normal log-p-harmonic mapping in the unit disk $\mathbb{D}$.

Proof. Let $F(z)=\sum_{k=1}^{p}|z|^{2(k-1)} G_{p-k+1}(z)$, for each $k \in\{1, \cdots, p\}$. We may represent the harmonic functions $G_{p-k+1}(z)=\log$ $g_{p-k+1}$ in series form as

$$
G_{p-k+1}(z)=\sum_{n=1}^{\infty} a_{j, p-k+1} z^{j}+\sum_{n=1}^{\infty} b_{j, p k+1}^{-} \bar{z}^{j}
$$

Then, $F=\log f$ is a polyharmonic mapping in $\mathbb{D}$. We know that

$$
\lambda_{f}(0)=\left\|f_{z}(0)|-| f_{\bar{z}}(0)\right\|=|f(0)||| F_{z}(0)|-| F_{\bar{z}}(0) \|,
$$

and $f(0)=1$, so it follows from $g_{p}(0)=\lambda_{f}(0)=1$, we have $G_{p}(0)=\lambda_{F}(0)-1=0$.

Obviously,

$$
\begin{aligned}
\left|G_{p-k+1}\right| & =\left|\log g_{p-k+1}\right|=|\log | g_{p-k+1}\left|+i \arg g_{p-k+1}\right| \\
& \leq|\log | g_{p-k+1}||+\pi,
\end{aligned}
$$

so we have

$$
\left|G_{p-k+1}\right| \leq \log M_{p-k+1}+\pi=\tilde{M}_{p-k+1} .
$$

In order to prove the normality of $f$, it follows from Theorem 6 that we have

$$
\left|F_{z}(z)+F_{\bar{z}}(z)\right| \leq S_{5}(r)
$$

where

$$
\begin{aligned}
S_{5}(r)= & \frac{4}{\pi\left(1-r^{2}\right)} \sum_{k=1}^{p-1} r^{2 k} \tilde{M}_{p-k}+\frac{8}{\pi(1-r)} \sum_{k=1}^{p-1} k \tilde{M}_{p-k} r^{2 k} \\
& +\frac{\Lambda_{p}^{2}-1}{\Lambda_{p}} \frac{r}{1-r}+\Lambda_{p} .
\end{aligned}
$$

So we obtain

$$
\left|\frac{f_{z}(z)}{f(z)}+\frac{f_{\bar{z}}(z)}{f(z)}\right| \leq S_{5}(r) .
$$

Differentiating $S_{5}(r)$, we have

$$
\begin{aligned}
S_{5}{ }^{\prime}(r)= & \sum_{k=1}^{p-1} \frac{4 M_{p-k}\left[2 k r^{2 k-1}\left(1-r^{2}\right)+2 r^{2 k+1}\right]}{\pi\left(1-r^{2}\right)^{2}} \\
& +\sum_{k=1}^{p-1} \frac{8 k \tilde{M}_{p-k}\left[2 k r^{2 k-1}(1-r)+r^{2 k}\right]}{\pi(1-r)^{2}}+\frac{\Lambda_{p}^{2}-1}{\Lambda_{p}(1-r)^{2}} .
\end{aligned}
$$

By elementary calculations, we get $S_{5}{ }^{\prime}(r)>0$. It follows that $S_{5}(r)$ is strictly increasing in $(0,1)$, and

$$
\lim _{r \rightarrow 0} S_{5}(r)=\Lambda_{p}, \quad \lim _{r \rightarrow 1} S_{5}(r)=+\infty
$$

It implies that $S_{5}(r)$ is bounded in $(0,1)$. Hence, there exists a finite value $m_{1}$ such that

$$
\left|\frac{f_{z}(z)}{f(z)}+\frac{f_{\bar{z}}(z)}{f(z)}\right| \leq m_{1}
$$

Finally, we consider any $z$ with $|z|=r_{3}$; then, we have

$$
\begin{aligned}
|\log f(z)|= & |F(z)|=\left.\left|\sum_{n=1}^{\infty}\left(a_{n, p} z^{n}+b_{n, p}^{-} \bar{z}^{n}\right)+\sum_{k=1}^{p-1}\right| z\right|^{2 k} G_{p-k}(z) \mid \\
\leq & \left|a_{1, p} z+b_{1, p}^{-} \bar{z}\right|+\left|\sum_{n=2}^{\infty}\left(a_{n, p} z^{n}+b_{n, p}^{-} \bar{z}^{n}\right)\right| \\
& +\left.\left|\sum_{k=1}^{p-1}\right| z\right|^{2 k} G_{p-k}(z) \mid \leq \Lambda_{p} r_{3}+\frac{\Lambda_{p}^{2}-1}{\Lambda_{p}} \sum_{n=2}^{\infty} \frac{r_{3}^{n}}{n} \\
& +\sum_{k=1}^{p-1} \frac{4 \tilde{M}_{p-k} r_{3}^{2 k+1}}{\pi\left(1-r_{3}\right)}=\sum_{k=1}^{p-1} \frac{4 \tilde{M}_{p-k} r_{3}^{2 k+1}}{\pi\left(1-r_{3}\right)} \\
& -\frac{\Lambda_{p}^{2}-1}{\Lambda_{p}}\left[r_{3}+\ln \left(1-r_{3}\right)\right]+\Lambda_{p} r_{3}=S_{6}\left(r_{3}\right) .
\end{aligned}
$$

Differentiating $S_{6}\left(r_{3}\right)$, have the following result

$$
\begin{aligned}
S_{6}{ }^{\prime}\left(r_{3}\right)= & \sum_{k=1}^{p-1} \frac{4 \tilde{M}_{p-k}(2 k+1)\left(1-r_{3}\right) r_{3}^{2 k}+r_{3}^{2 k+1}}{\pi\left(1-r_{3}\right)^{2}} \\
& +\frac{\Lambda_{p}^{2}-1}{\Lambda_{p}\left(1-r_{3}\right)}+\frac{1}{\Lambda_{p}}
\end{aligned}
$$

It is not difficult to verify that $S_{6}{ }^{\prime}\left(r_{3}\right)>0$, which means that $S_{6}\left(r_{3}\right)$ is strictly increasing. It is also easily seen that $S_{6}$ $\left(r_{3}\right)$ is bounded in $(0,1)$. Hence, there is a finite value $m_{2}$ such that 


$$
|\log f(z)| \leq m_{2}
$$

Applying Lemma 5 and (63), (66), we obtain $f$ is normal in $\mathbb{D}$. The proof of Theorem 8 is complete.

\section{Data Availability}

The data used to support the findings of this study are included with the article.

\section{Conflicts of Interest}

We declare that we have no competing interests.

\section{Acknowledgments}

The first author would like to thank the Department of Mathematics, University of Houston, USA, for providing a good environment during the preparation of this work. The corresponding authors would like to thank the visiting scholar program of Shiing-Shen Chern Institute of Mathematics at Nankai University. The authors are also thankful to the referees for their invaluable comments and suggestions. This research was supported by the National Natural Science Foundation of China under Grant 11701111, 12031003, and 11771347.

\section{References}

[1] P. Lappan, "Some results on harmonic normal functions," Mathematische Zeitschrift, vol. 90, no. 2, pp. 155-159, 1965.

[2] R. Aulaskari and P. Lappan, "An integral condition for harmonic normal functions," Complex Variables, Theory and Application: An International Journal, vol. 23, no. 3-4, pp. 213-219, 1993.

[3] X. Chen and A. Fang, "A schwarz-pick inequality for harmonic quasiconformal mappings and its applications," Journal of Mathematical Analysis and Applications, vol. 369, no. 1, pp. 22-28, 2010.

[4] X. Chen and T. Qian, "Estimation of hyperbolically partial derivatives of $\rho$-harmonic quasiconformal mappings and its applications," Complex Variables and Elliptic Equations, vol. 60, no. 6, pp. 875-892, 2015.

[5] X. Chen and Y. Que, "Quasiconformal extensions of harmonic mappings with a complex parameter," Journal of the Australian Mathematical Society, vol. 102, no. 3, pp. 307-315, 2017.

[6] S. Chen and S. Ponnusamy, "John Disks and k-quasiconformal harmonic mappings," The Journal of Geometric Analysis, vol. 27, no. 2, pp. 1468-1488, 2017.

[7] S. Chen, S. Ponnusamy, and X. Wang, "Recent results on harmonic and p-harmonic mappings," Journal of Analysis, vol. 18, pp. 99-128, 2011.

[8] S. Chen and J. Zhu, "Schwarz type Lemmas and a Landau type theorem of functions satisfying the biharmonic equation," Bulletin des Sciences Mathématiques, vol. 154, pp. 36-63, 2019.

[9] Z. Liu and S. Ponnusamy, "Bohr radius for subordination and k-quasiconformal harmonic mappings," Bulletin of the Malaysian Mathematical Sciences Society, vol. 42, no. 5, pp. 21512168, 2019.
[10] Z. Liu and S. Ponnusamy, "Some properties of univalent logharmonic mappings," Filomat, vol. 32, no. 15, pp. 52755288, 2018.

[11] R. Hernández and M. J. Martín, "Stable geometric properties of analytic and harmonic functions," Mathematical Proceedings of the Cambridge Philosophical Society, vol. 155, no. 2, pp. 343-359, 2013.

[12] S. M. S. Kanas, S. Maharana, and J. K. Prajapat, "Norm of the pre-Schwarzian derivative, Bloch's constant and coefficient bounds in some classes of harmonic mappings," Journal of Mathematical Analysis and Applications, vol. 474, no. 2, pp. 931-943, 2019.

[13] J. K. P. S. Ponnusamy and A. S. Kaliraj, "Uniformly starlike and uniformly convex harmonic, mappings," Journal of Analysis, vol. 23, pp. 121-129, 2015.

[14] F. Colonna, "The Bloch constant of bounded harmonic mappings," Indiana University Mathematics Journal, vol. 38, no. 4, pp. 829-840, 1989.

[15] H. Lewy, "On the non-vanishing of the Jacobian in certain one-to-one mappings," Bulletin of the American Mathematical Society, vol. 42, no. 10, pp. 689-693, 1936.

[16] S. Chen, S. Ponnusamy, and X. Wang, "Bloch constant and Landau's theorem for planar p-harmonic mappings," Journal of Mathematical Analysis and Applications, vol. 373, no. 1, pp. 102-110, 2011.

[17] M. Dorff and M. Nowak, "Landau's theorem for planar harmonic mappings," Computational Methods and Function Theory, vol. 4, no. 1, pp. 151-158, 2004.

[18] M.-S. Liu, "Landau's theorems for biharmonic mappings," Complex Variables and Elliptic Equations, vol. 53, no. 9, pp. 843-855, 2008.

[19] X. Bai and M. Liu, "Landau-type theorems of polyharmonic mappings and log-p-harmonic mappings," Complex Analysis and Operator Theory, vol. 13, no. 2, pp. 321-340, 2019.

[20] Y. Jiang, Z. Liu, and S. Ponnusamy, "Univalent harmonic mappings and lift to the minimal surfaces," Lobachevskii Journal of Mathematics, vol. 40, no. 9, pp. 1295-1312, 2019.

[21] M.-S. Liu, "Landau's theorem for planar harmonic mappings," Computers \& Mathematics with Applications, vol. 57, no. 7, pp. 1142-1146, 2009.

[22] M. Liu and Z. Liu, "Landau-type theorems for p-harmonic mappings or log-p-harmonic mappings," Applicable Analysis, vol. 93, no. 11, pp. 2462-2477, 2014.

[23] Z. Mao, S. Ponnusamy, and X. Wang, "Schwarzian derivative and Landau's theorem for logharmonic mappings," Complex Variables and Elliptic Equations, vol. 58, no. 8, pp. 10931107, 2013.

[24] P. Li and X. Wang, "Landau's theorem for log-p-harmonic mappings," Applied Mathematics and Computation, vol. 218, pp. 4806-4812, 2012.

[25] M. Liu, "Estimates on Bloch constants for planar harmonic mappings," Science in China Series A: Mathematics, vol. 52, no. 1, pp. 87-93, 2009. 